\title{
Constraining very-high-energy and optical emission from FRB 121102 with the MAGIC telescopes
}

MAGIC Collaboration: V. A. Acciari, ${ }^{1}$ S. Ansoldi, ${ }^{2,3}$ L. A. Antonelli, ${ }^{4}$

A. Arbet Engels, ${ }^{5}$ C. Arcaro, ${ }^{6}$ D. Baack,${ }^{7}$ A. Babić, ${ }^{8}$ B. Banerjee, ${ }^{9}$ P. Bangale,${ }^{10}$

U. Barres de Almeida, ${ }^{10} \dagger$ J. A. Barrio, ${ }^{11}$ J. Becerra González, ${ }^{1}$ W. Bednarek, ${ }^{12}$

E. Bernardini, ${ }^{6,13,14}$ A. Berti, ${ }^{3,15}$ J. Besenrieder, ${ }^{10}$ W. Bhattacharyya, ${ }^{13}$ C. Bigongiari, ${ }^{4}$ A. Biland, ${ }^{5}$ O. Blanch,${ }^{16}$ G. Bonnoli, ${ }^{17}$ R. Carosi, ${ }^{18}$ G. Ceribella, ${ }^{10}$ A. Chatterjee, ${ }^{9}$ S. M. Colak, ${ }^{16}$ P. Colin, ${ }^{10}$ E. Colombo, ${ }^{1}$ J. L. Contreras, ${ }^{11}$ J. Cortina,,${ }^{16}$ S. Covino, ${ }^{4}$ P. Cumani, ${ }^{16}$ V. D'Elia, ${ }^{4}$ P. Da Vela,${ }^{17}$ F. Dazzi,${ }^{4}$ A. De Angelis, ${ }^{6}$ B. De Lotto, ${ }^{3}$ M. Delfino, ${ }^{16,19}$ J. Delgado, ${ }^{16,19}$ F. Di Pierro,${ }^{6}$ A. Domínguez, ${ }^{11}$ D. Dominis Prester, ${ }^{8}$ D. Dorner, ${ }^{20}$ M. Doro, ${ }^{6}$ S. Einecke,${ }^{7}$ D. Elsaesser ${ }^{7}$ V. Fallah Ramazani, ${ }^{21}$ A. Fattorini, ${ }^{7}$ A. Fernández-Barral, ${ }^{6}$ G. Ferrara,${ }^{4}$ D. Fidalgo, ${ }^{11}$ L. Foffano, ${ }^{6}$ M. V. Fonseca, ${ }^{11}$

L. Font, ${ }^{22}$ C. Fruck, ${ }^{10}$ S. Gallozzi, ${ }^{4}$ R. J. García López, ${ }^{1}$ M. Garczarczyk, ${ }^{13}$ M. Gaug ${ }^{22}$ P. Giammaria, ${ }^{4}$ N. Godinović, ${ }^{8}$ D. Guberman, ${ }^{16}$ D. Hadasch, ${ }^{2}$ A. Hahn, ${ }^{10}$ T. Hassan, ${ }^{16 \star}$ J. Herrera, ${ }^{1}$ J. Hoang, ${ }^{1,11 \star}$ D. Hrupec, ${ }^{8}$ S. Inoue ${ }^{\oplus},{ }^{2 \star}$ K. Ishio, ${ }^{10}$ Y. Iwamura, ${ }^{2}$ H. Kubo, ${ }^{2}$ J. Kushida, ${ }^{2}$ D. Kuveždić, ${ }^{8}$ A. Lamastra,${ }^{4}$ D. Lelas, ${ }^{8}$ F. Leone, ${ }^{4}$ E. Lindfors, ${ }^{21}$

S. Lombardi, ${ }^{4}$ F. Longo, ${ }^{3,15}$ M. López, ${ }^{11}$ A. López-Oramas, ${ }^{1}$ C. Maggio, ${ }^{22}$

P. Majumdar, ${ }^{9}$ M. Makariev, ${ }^{23}$ G. Maneva, ${ }^{23}$ M. Manganaro, ${ }^{1}$ K. Mannheim,,${ }^{20}$ L. Maraschi, ${ }^{4}$ M. Mariotti, ${ }^{6}$ M. Martínez, ${ }^{16}$ S. Masuda, ${ }^{2}$ D. Mazin,,${ }^{2,10}$ M. Minev, ${ }^{23}$ J. M. Miranda, ${ }^{17}$ R. Mirzoyan, ${ }^{10}$ E. Molina,${ }^{24}$ A. Moralejo, ${ }^{16}$ V. Moreno, ${ }^{22}$ E. Moretti, ${ }^{16}$ V. Neustroev, ${ }^{21}$ A. Niedzwiecki, ${ }^{12}$ M. Nievas Rosillo, ${ }^{11}$ C. Nigro, ${ }^{13}$ K. Nilsson, ${ }^{21}$ D. Ninci,${ }^{16}$ K. Nishijima, ${ }^{2}$ K. Noda,${ }^{2}$ L. Nogués, ${ }^{16}$ S. Paiano, ${ }^{6}$ J. Palacio, ${ }^{16}$ D. Paneque, ${ }^{10}$ R. Paoletti, ${ }^{17}$ J. M. Paredes,${ }^{24}$ G. Pedaletti, ${ }^{13}$ P. Peñil,,${ }^{11}$ M. Peresano, ${ }^{3}$ M. Persic,${ }^{3,25}$ P. G. Prada Moroni, ${ }^{18}$ E. Prandini, ${ }^{6}$ I. Puljak, ${ }^{8}$ J. R. Garcia, ${ }^{10}$ W. Rhode, ${ }^{7}$ M. Ribó ${ }^{24}$ J. Rico, ${ }^{16}$ C. Righi, ${ }^{4}$ A. Rugliancich, ${ }^{17}$ L. Saha,${ }^{11}$ T. Saito,${ }^{2}$ K. Satalecka, ${ }^{13}$ T. Schweizer, ${ }^{10}$ J. Sitarek, ${ }^{12}$ I. Šnidarić, ${ }^{8}$ D. Sobczynska, ${ }^{12}$ A. Somero, ${ }^{1}$ A. Stamerra ${ }^{4}$ M. Strzys, ${ }^{10}$ T. Surić,${ }^{8}$ F. Tavecchio, ${ }^{4}$ P. Temnikov, ${ }^{23}$ T. Terzić, ${ }^{8}$ M. Teshima,${ }^{2,10}$ N. Torres-Albà, ${ }^{24}$ S. Tsujimoto,${ }^{2}$ G. Vanzo, ${ }^{1}$ M. Vazquez Acosta, ${ }^{1}$ I. Vovk, ${ }^{10}$ J. E. Ward,$^{16}$ M. Will, ${ }^{10}$ D. Zarić, ${ }^{8}$ Collaborators: B. Marcote ${ }^{\odot},{ }^{26}$ L. G. Spitler, ${ }^{27}$ J. W. T. Hessels, ${ }^{28,29}$ K. Kashiyama, ${ }^{30}$ K. Murase, ${ }^{31}$ V. Bosch-Ramon, ${ }^{24}$ D. Michilli ${ }^{28,29}$ and A. Seymour ${ }^{32}$

Affiliations are listed at the end of the paper

Accepted 2018 September 2. Received 2018 August 23; in original form 2018 May 14 


\section{ABSTRACT}

Fast radio bursts (FRBs) are bright flashes observed typically at GHz frequencies with millisecond duration, whose origin is likely extragalactic. Their nature remains mysterious, motivating searches for counterparts at other wavelengths. FRB 121102 is so far the only source known to repeatedly emit FRBs and is associated with a host galaxy at redshift $z \simeq 0.193$. We conducted simultaneous observations of FRB 121102 with the Arecibo and MAGIC telescopes during several epochs in 2016-2017. This allowed searches for millisecond time-scale burst emission in very-high-energy (VHE) gamma-rays as well as the optical band. While a total of five FRBs were detected during these observations, no VHE emission was detected, neither of a persistent nature nor burst-like associated with the FRBs. The average integral flux upper limits above $100 \mathrm{GeV}$ at 95 percent confidence level are $6.6 \times 10^{-12}$ photons $\mathrm{cm}^{-2} \mathrm{~s}^{-1}$ (corresponding to luminosity $L_{\mathrm{VHE}} \lesssim 10^{45} \mathrm{erg} \mathrm{s}^{-1}$ ) over the entire observation period, and $1.2 \times 10^{-7}$ photons $\mathrm{cm}^{-2} \mathrm{~s}^{-1}\left(L_{\mathrm{VHE}} \lesssim 10^{49} \mathrm{erg} \mathrm{s}^{-1}\right)$ over the total duration of the five FRBs. We constrain the optical $U$-band flux to be below $8.6 \mathrm{mJy}$ at $5 \sigma$ level for 1-ms intervals around the FRB arrival times. A bright burst with $U$-band flux 29 mJy and duration $\sim 12 \mathrm{~ms}$ was detected $4.3 \mathrm{~s}$ before the arrival of one FRB. However, the probability of spuriously detecting such a signal within the sampled time space is 1.5 per cent (2.2, post-trial), i.e. consistent with the expected background. We discuss the implications of the obtained upper limits for constraining FRB models.

Key words: radiation mechanisms: non-thermal-methods: data analysis - methods: observational-gamma-rays: general.

\section{INTRODUCTION}

Fast radio bursts (FRBs) are astrophysical phenomena that exhibit bright, transient pulses of millisecond duration, typically at $\mathrm{GHz}$ frequencies. First discovered by Lorimer et al. (2007), around 30 such events have been found to date ${ }^{1}$ (Thornton et al. 2013; Petroff et al. 2016). The dispersion measures (DM) observed in FRBs imply intervening column densities of free electrons that are significantly larger than those expected from the Galactic interstellar medium, strongly suggesting their extragalactic origin (cf. Ioka 2003; Inoue 2004). However, the nature of FRBs still remains uncertain, mainly because the single-dish radio telescopes used to detect most of these FRBs have localization capabilities that are insufficient for unambiguous identification with counterparts at other wavelengths. A wide variety of theoretical models has been proposed to explain FRBs (see e.g. Rane \& Lorimer 2017; Katz 2018 for reviews).

Among the FRB population, only one, FRB 121102, is currently known to exhibit repeating bursts (Spitler et al. 2014, 2016; Scholz et al. 2016). Its repetitive nature allowed the localization of the source to sub-arcsecond precision, and the discovery of persistent associated sources in the optical and radio bands (Chatterjee et al. 2017; Marcote et al. 2017). It was found to be located in a low-metallicity star-forming region of a dwarf galaxy with $m_{r}$ $=25.1 \pm 0.1 \mathrm{mag}$ at a redshift of $z \simeq 0.193$ (Bassa et al. 2017; Tendulkar et al. 2017). The source is localized within a projected separation of $40 \mathrm{pc}$ from a compact $(\lesssim 0.7 \mathrm{pc})$ and persistent radio source (Marcote et al. 2017).

These findings prompted searches for counterparts of FRB 121102 at other wavelengths. Hardy et al. (2017) conducted simultaneous radio and optical observations. Out of a total of 13 radio bursts detected, no significant optical bursts were found above a flux density of $0.33 \mathrm{mJy}$ at $767 \mathrm{~nm}$, corresponding to a fluence limit of $46 \mathrm{mJy} \mathrm{ms}$. Scholz et al. (2017) performed simultaneous radio

\footnotetext{
${ }^{1}$ See the online FRB Catalogue for a list of currently known FRBs:http:
} //www.astronomy.swin.edu.au/pulsar/frbcat/ and X-ray observations. They detected 12 radio bursts, but no X-ray bursts were found in coincidence or at any other epoch, implying $5 \sigma$ fluence upper limits of $3 \times 10^{-11}$ and $5 \times 10^{-10} \mathrm{erg} \mathrm{cm}^{-2}$ at 0.5 and $10 \mathrm{keV}$, respectively. No persistent X-ray emission at the position of FRB 121102 was detected. The authors also analysed Fermi-GBM data during the epochs of those 12 radio bursts, placing $5 \sigma$ fluence upper limits of $4 \times 10^{-9} \mathrm{erg} \mathrm{cm}^{-2}\left(5 \times 10^{47} \mathrm{erg}\right.$ in time-integrated energy at the distance of FRB 121102) in the 10-100 keV energy range. Zhang \& Zhang (2017) analysed the eight-year Fermi-LAT data to search for persistent gamma-ray emission. No evidence of emission was found, implying an upper limit of $4 \times 10^{44} \mathrm{erg} \mathrm{s}^{-1}$ on the GeV-band luminosity. Bird et al. (2017) constrained the persistent very-high-energy (VHE; $\gtrsim 0.1 \mathrm{TeV}$ ) emission with VERITAS, setting differential upper limits of $5.2 \times 10^{-12}$ and $4.0 \times 10^{-11} \mathrm{~cm}^{-2} \mathrm{~s}^{-1} \mathrm{TeV}^{-1}$ at their energy thresholds of 0.2 and $0.15 \mathrm{TeV}$ (assuming power-law spectra with indices -2 and -4 , respectively).

Various scenarios have been proposed to explain FRB 121102 and the associated persistent radio source. A widely discussed class of FRB progenitors involve neutron stars that are either rotationally powered (e.g. Connor, Sievers \& Pen 2016; Cordes \& Wasserman 2016; Lyutikov, Burzawa \& Popov 2016) or magnetically powered (e.g. Popov \& Postnov 2013; Lyubarsky 2014). Pulsar wind nebulae driven by such neutron stars had been predicted as persistent radio counterparts (Murase, Kashiyama \& Mészáros 2016). Kashiyama $\&$ Murase (2017) showed that a young (10-100 yr old) neutron star powering a pulsar wind nebula inside a supernova remnant could be responsible for FRB 121102. The location of FRB 121102 inside a low-metallicity star-forming region (Tendulkar et al. 2017) may point to a magnetar, as such environments are similar to the hosts of hydrogen-poor super-luminous supernovae, whose progenitors could be young magnetars (Lunnan et al. 2014). On the other hand, Waxman (2017) suggested a self-consistent scenario for both the bursts and the persistent source, in which the associated nebula is surrounded by low-mass ejecta rather than massive ejecta that is often expected for magnetar progenitors (e.g. Tendulkar, Kaspi \& Patel 2016; Kashiyama \& Murase 2017). 
In a magnetar scenario, quasi-simultaneous $\mathrm{X}$-ray to $\mathrm{MeV}$ gamma-ray bursts could be produced analogously to those observed in short bursts from Galactic magnetars, with a X-ray-to-radio fluence ratio $\sim 10^{4}$ (Lyutikov 2002). VHE gamma-ray flashes correlated with FRBs have also been predicted, arising from the interaction of ultra-relativistic outflows triggered by magnetic dissipation with the ambient nebula, with VHE-to-radio fluence ratios $\sim 10^{5}$ $10^{6}$ (Lyubarsky 2014; Murase et al. 2016). Such VHE emission is possible in certain generic conditions, where an FRB progenitor like a young neutron star or a young white dwarf is naturally surrounded by a hot nebula. If magnetic bursts occur inside the bubble, pre-existing high-energy particles accelerated around the wind termination radius may be accelerated further by the impulsive energy injection into the nebula (Murase et al. 2016). The consequent VHE emission may be detectable when the external shock is strong enough. Such a scheme has also been explored in the synchrotron maser model for FRB emission (Lyubarsky 2014). On the other hand, if the FRBs were caused by coherent curvature radiation, most of the emission may be concentrated in the radio domain, without obvious counterparts at other wavelengths (Ghisellini \& Locatelli 2017). If FRBs are produced via forced reconnection of magnetic fields near the surface of magnetars, Kumar, Lu \& Bhattacharya (2017) predicts ms bursts up to optical wavelengths, although independently of FRBs and with a lower burst rate.

An alternative scenario invokes a relativistic jet ejected by a massive black hole $(\mathrm{BH})$ for the origin of both the FRBs and the persistent radio source (Vieyro et al. 2017). The luminosity and compactness of the latter may be consistent with a $\mathrm{BH}$ with mass $10^{4}-10^{6} \mathrm{M}_{\odot}$ (Marcote et al. 2017). Vieyro et al. (2017) suggest that detectable high-energy emission associated with FRBs may occur on time-scales of seconds to minutes under certain conditions.

Magnetars can also coexist with a massive $\mathrm{BH}$ in the central regions of galaxies (e.g. Pen \& Connor 2015; Cordes \& Wasserman 2016), in which case the former may be responsible for the FRBs and the latter for the persistent radio source. Such systems could be analogous to the magnetars known to exist in the Galactic Center, but with more extreme conditions (Pen \& Connor 2015), possibly even interacting with each other (Zhang 2018). The recent discovery of extremely large and variable Faraday rotation of linearly polarized radiation of the bursts from FRB 121102 may be consistent with such environments (Michilli et al. 2018).

To summarize the current knowledge, the progenitors and mechanisms producing FRBs are not well understood, and a variety of predictions have been made for associated counterparts across the electromagnetic spectrum. New and deeper constraints at other wavelengths are necessary to clarify their origin. In this paper, we present optical and VHE observations of FRB 121102 simultaneous with radio observations. The detection of radio bursts during these observations allows us to constrain optical and VHE counterparts correlated in time.

Section 2 describes our simultaneous radio, optical, and VHE observations and the data analysis methods. Section 3 presents the results of the observations. Section 4 discusses the constraints on the multiwavelength emission of FRB 121102 and the implications. Section 5 concludes this work.

\section{INSTRUMENTS, OBSERVATIONS, AND ANALYSIS}

In September 2016, we started a campaign of simultaneous observations with the MAGIC (Major Atmospheric Gamma Imaging Cherenkov) telescopes and the Arecibo radio telescope. Observa- tions in VHE gamma-rays and in the optical band were carried out with MAGIC, making use of the central pixel installed on the MAGIC II camera (Lucarelli et al. 2008). We describe below the radio, optical, and VHE observations and the data analysis methods.

\subsection{Arecibo radio observations}

Radio observations of FRB 121102 were conducted with the 305-m William E. Gordon Telescope at the Arecibo Observatory at a central frequency of $1.38 \mathrm{GHz}$. We made use of the Puerto-Rican Ultimate Pulsar Processing Instrument (PUPPI) together with the single-pixel $L$-band wide receiver, which provide a total bandwidth of $800 \mathrm{MHz}$ and a usable bandwidth of $\sim 600 \mathrm{MHz}$ (due to radio frequency interference removal). The data were coherently de-dispersed to $\mathrm{DM}=$ $557 \mathrm{pc} \mathrm{cm}^{-3}$ (Spitler et al. 2014) to remove the dispersive smearing of the burst widths with a time resolution of $10.24 \mathrm{~s}$.

A total of five radio bursts from FRB 121102 were detected with a high significance during the simultaneous MAGIC and Arecibo observations using analogue methods as in Spitler et al. (2014). We list these bursts and the MAGIC observing conditions at their times of arrival (TOAs) in Table 1. We note that the listed Arecibo TOAs refer to the topocentric times on site at the top of the observed band $(1.73 \mathrm{GHz})$. TOAs at the MAGIC site have been corrected to infinite frequency considering the de-dispersion of the signal (776.4 ms) and also corrected for the different expected topocentric times (correction smaller than $10 \mathrm{~ms}$, varying between the different FRBs).

\subsection{MAGIC observations and data analysis}

The MAGIC telescope system consists of two 17-m imaging atmospheric Cherenkov telescopes, located at the Roque de los Muchachos Observatory on the island of La Palma, Canary Islands.

\subsubsection{MAGIC stereoscopic observations}

Stereoscopic observations with MAGIC provide an integral sensitivity of $0.66 \pm 0.03$ percent of the Crab Nebula flux above 220 $\mathrm{GeV}$ in $50 \mathrm{~h}$ of observation, and allow the measurement of photons in the energy range from $50 \mathrm{GeV}$ to above $50 \mathrm{TeV}$ (Aleksić et al. 2016b).

Observations of FRB 121102, taken up to 60 deg in zenith angle, were carried out in $\mathrm{ON}$ mode (i.e. with the source always located at the centre of the field of view) to allow simultaneous data taking in the optical range with the central pixel (see Section 2.2.2). The source was observed during 14 nights (between September 2016 and September 2017), with a total of $22 \mathrm{~h}$ of data surviving quality cuts, of which $8.9 \mathrm{~h}$ were simultaneous with Arecibo. MAGIC observing conditions during the five Arecibo TOAs were excellent, with atmospheric transmission and zenith angles shown in Table 1.

The VHE data analysis presented here was carried out using standard MAGIC analysis software (Zanin et al. 2013). Integral and differential flux upper limits were computed as in Rolke, López \& Conrad (2005) assuming a 30 percent systematic uncertainty on the efficiency. Given that $\mathrm{ON}$-mode observations do not allow to use the standard background evaluation methods (simultaneous background using reflected regions, see Aleksić et al. 2016b), the background was extracted from OFF data samples collected under similar conditions (mainly zenith angle and night-sky background level).

For the search of millisecond-time-scale VHE emission described in Section 3.2, integral flux upper limits were calculated assuming 
Table 1. FRBs detected by Arecibo during the campaign, together with the observing conditions for MAGIC at the corresponding epochs. The reported aerosol transmission refers to the atmospheric optical depth relative to a standard dark night. The radio peak brightnesses of the Arecibo bursts have been estimated via the radiometer equation with an uncertainty of $\sim 20$ per cent (see Scholz et al. 2016).

\begin{tabular}{|c|c|c|c|c|c|c|c|}
\hline $\begin{array}{l}\text { MJD (Arecibo) } \\
\text { (d) }\end{array}$ & $\begin{array}{c}\mathrm{DM} \\
\left(\mathrm{pc} \mathrm{cm} \mathrm{cm}^{-3}\right)\end{array}$ & $\begin{array}{l}\text { Duration } \\
\quad(\mathrm{ms})\end{array}$ & $\begin{array}{l}\text { Peak brightness } \\
\text { (Jy) }\end{array}$ & $\begin{array}{c}\text { Significance (Arecibo) } \\
(\sigma)\end{array}$ & $\begin{array}{c}\text { MJD (MAGIC site) } \\
\text { (d) }\end{array}$ & $\begin{array}{c}\text { Aerosol } \\
\text { transmission }\end{array}$ & $\begin{array}{c}\mathrm{Zd} \\
(\mathrm{deg})\end{array}$ \\
\hline 57799.98317566 & 562 & 5.73 & 1.4 & 32.17 & 57799.98316670 & 0.96 & 33 \\
\hline 57806.96425078 & 562 & 2.46 & 1.6 & 38.59 & 57806.96424183 & 0.96 & 33 \\
\hline 57806.98472905 & 561 & 3.69 & 1.5 & 35.21 & 57806.98472011 & 0.96 & 40 \\
\hline 57808.00278585 & 563 & 3.69 & 0.79 & 18.73 & 57808.00277693 & 0.96 & 46 \\
\hline 57814.94698520 & 560 & 1.15 & 0.47 & 11.13 & 57814.94697625 & 0.96 & 35 \\
\hline
\end{tabular}

that the expected number of photons within a 10 -ms time window follows a Poisson distribution with no expected background [see for instance table 39.3 in Patrignani \& Particle Data Group (2016)]. A toy Monte Carlo simulation was performed to estimate the flux upper limit under the assumption of a 30 per cent Gaussian systematic uncertainty on the mean.

\subsubsection{MAGIC central pixel}

The MAGIC telescopes are able to operate simultaneously as both VHE and optical telescopes, with excellent sensitivity in the two regimes. Optical observations are performed using only one pixel within one of the MAGIC cameras, namely the central pixel, which covers a $0.1 \mathrm{deg}$ field of view. It consists of a fully modified photosensor-to-readout chain at the centre of the MAGIC-II telescope camera, increasing the bandwidth of the central pixel DC branch from $8 \mathrm{~Hz}$ to over $3 \mathrm{kHz}$ (Hassan et al. 2017). After the upgrade carried out in 2011-2012 (Aleksić et al. 2016a), MAGIC is able to detect the optical pulsations of the Crab Pulsar with observation times shorter than $10 \mathrm{~s}$ (Hassan et al. 2017). By studying the dispersion within the off-source data and making use of the well known flux and phaseogram of the Crab Pulsar, the MAGIC central pixel is able to detect isolated 1-ms optical flashes as faint as $\sim 8 \mathrm{mJy}(13.4 \mathrm{mag}$ ) with maximum sensitivity at $350 \mathrm{~nm}$ (Borla Tridon et al. 2009; Hassan et al. 2017). The central pixel data exhibits some low frequency noise, mainly caused by surrounding camera components. In order to improve sensitivity to $1-10 \mathrm{~ms}$ non-periodic optical pulses, averaging filters of variable integration length (1-10 ms) were applied to denoise the central pixel data with a standard sampling rate of $10 \mathrm{kHz}$. At each point, the uncertainty is taken as the standard deviation of each $1-10 \mathrm{~ms}$ window.

Various external light sources such as meteors, car flashes, satellites, and space debris are able to produce fast optical pulses, constituting backgrounds in searching for optical counterparts to FRBs. Several methods to identify these events have been developed and implemented for the data analysis presented here, mainly involving the use of average pixel DC current reports that are stored every second during observations. Identifying variations in the average camera currents on time-scales of seconds to minutes efficiently removes slowly varying optical signals (e.g. car flashes or satellites). However, after the use of these filters, an irreducible background still remains, mainly produced by faint meteors passing through the field of view of the central pixel, producing signals lasting about 5 to $20 \mathrm{~ms}$.

The frequency of these background events was studied to allow the calculation of the significance of a hypothetical nonsimultaneous optical pulse that may precede or follow the radio bursts. Within each off-source data run, after applying a 1-ms av-
Table 2. Upper limits on the persistent VHE emission of FRB 121102 in terms of integral flux above $E_{0}$, assuming power-law spectra with $\Gamma=2$ and $\Gamma=4$.

\begin{tabular}{lcc}
\hline $\begin{array}{l}E_{0} \\
{[\mathrm{GeV}]}\end{array}$ & $\begin{array}{c}\text { Int. flux UL }(\Gamma=2) \\
{\left[10^{-12} \mathrm{~cm}^{-2} \mathrm{~s}^{-1}\right]}\end{array}$ & $\begin{array}{c}\text { Int. flux UL }(\Gamma=4) \\
{\left[10^{-12} \mathrm{~cm}^{-2} \mathrm{~s}^{-1}\right]}\end{array}$ \\
\hline 100 & 6.6 & 12 \\
400 & 1.7 & 1.9 \\
1000 & 0.37 & 0.33 \\
\hline
\end{tabular}

eraging filter and the selection criteria described above, any signal exceeding 5 times the standard deviation of the average voltage was classified as a possible optical background pulse. Even if the background rate is low (frequency between $10^{-2}-10^{-5} \mathrm{~Hz}$, decreasing with brightness), it hinders the possibility of associating with high confidence an optical burst that is not precisely simultaneous with a given FRB.

The central pixel sensitivity and timing precision was tested every night of FRB 121102 observations by dedicating 5 min to observe the Crab Pulsar. To convert the central pixel output voltage to the corresponding optical absolute magnitude, an empirical expression was derived by fitting the measured phaseogram of the Crab Pulsar to its well known flux profile, as done in Hassan et al. (2017). The magnitude in the $U$ band measured by the central pixel can be expressed as

$m_{\mathrm{U}}=m_{\mathrm{U}, \mathrm{CP}}-2.5 \log _{10}\left(2.15 \times 10^{3} \mathrm{~V}-2.28 \times 10^{2}\right)$,

where $m_{\mathrm{U}, \mathrm{CP}}$ is the average optical magnitude of the Crab Pulsar in the $U$ band ( $\sim 16.9 \mathrm{mag}$ ) and $V$ is the output voltage in volts.

\section{RESULTS}

Given the large variety of predictions available for counterparts across the electromagnetic spectrum, several kinds of searches have been performed with the data sample described in Section 2.2. We searched for millisecond-time-scale burst emission associated with the FRBs detected by Arecibo, both in the optical and VHE range, as well as persistent VHE gamma-ray emission as in Bird et al. (2017).

\subsection{Persistent VHE emission}

No persistent VHE gamma-ray emission was detected from FRB 121102. Assuming a power-law spectrum with photon index $\Gamma$, integral flux upper limits (ULs) were calculated above 100, 400, and $1000 \mathrm{GeV}$ at 95 percent confidence level. These results are shown in Table 2. For the specific case of $\Gamma=2$ assumed for each energy bin, differential flux upper limits are shown in Fig. 1. 


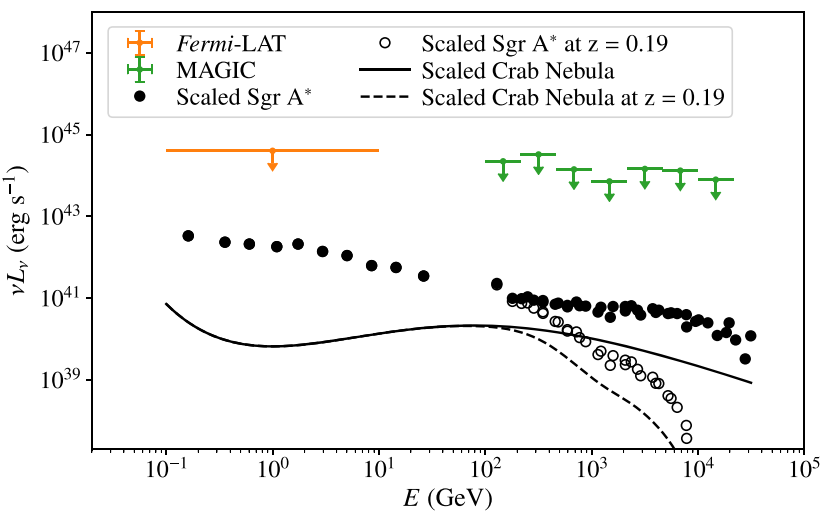

Figure 1. Upper limits in luminosity for the persistent gamma-ray emission of FRB 121102 from MAGIC (95 per cent confidence level, assuming an intrinsic power-law spectrum with $\Gamma=2$ and 30 per cent overall systematic uncertainty). Limits from Fermi-LAT (Zhang \& Zhang 2017) are also shown. The black curve and filled circles represent, respectively, the SEDs of the Crab Nebula (Meyer, Horns \& Zechlin 2010) and Sgr A* (Abdo et al. 2009; Aharonian et al. 2009), scaled by factors of $4 \times 10^{5}$ and $2 \times 10^{6}$ to match the observed radio luminosity of the persistent counterpart of FRB 121102 (see Section 4.1). The dashed black curve and empty circles show the effect of gamma-ray attenuation from $z=0.19$ due to the EBL, following Domínguez et al. (2011)

\subsection{Millisecond-time-scale VHE emission}

Fixing a time window of $10 \mathrm{~ms}$ centred around the radio burst TOAs and using custom analysis cuts (on size, Hadronness, and $\theta^{2}$; see Aleksić et al. 2016b) that are optimized to maximize sensitivity for a 10-ms signal, no gamma-like events are found within any of these windows above $100 \mathrm{GeV}$. Since the background rate during such time intervals is negligible with $<10^{-2}$, the resulting UL (95 per cent $\mathrm{CL}$, adding 30 per cent systematic uncertainty to the Poisson mean) for each FRB corresponds to 3.56 events (see Section 2.2.1). The corresponding integral flux upper limits for individual FRBs in different energy ranges are shown in Table 3, assuming a powerlaw spectrum with two different indices, $\Gamma=2$ and $\Gamma=4$. A combined integral flux upper limit is also derived by stacking the data around the five FRB TOAs, over a duration of $5 \times 10 \mathrm{~ms}$.

Given that the properties of FRBs are unknown, offsets in the arrival times of the burst emission at radio and higher frequency are possible. A blind search for non-simultaneous VHE bursts was also performed. In this case, due to the large number of trials, the sensitivity worsens significantly. A (non-overlapping) sliding 10-ms window sampling the arrival time of all events surviving analysis cuts was used through the whole data set $\left(1.2 \times 10^{5}\right.$ trials per 20 -min run).

No hint of VHE bursts was found, for any offset up to an hour with respect to the Arecibo TOAs. The minimum flux of VHE photons detectable by this blind search was calculated from the joint probability density function of all gamma-like events observed, corrected by the total number of trials performed. We conclude that a single 10 -ms burst with a flux of $8.2 \times 10^{-5} \mathrm{~cm}^{-2} \mathrm{~s}^{-1}$ above 100 $\mathrm{GeV}$ (equivalent to an isotropic luminosity of $L_{\mathrm{VHE}} \sim 10^{52} \mathrm{erg} \mathrm{s}^{-1}$ ) would have been firmly detected $(S>5 \sigma)$, assuming power-law spectra with $\Gamma=2$.

\subsection{Millisecond-time-scale optical emission}

As described in Section 2.2.2, observations were carried out in the optical $U$-band using the MAGIC central pixel. As shown in Fig. 2,
Table 3. Upper limits on VHE burst emission of FRB 121102, in terms of integral flux above $E_{0}$ over $10 \mathrm{~ms}$ intervals around the TOAs of each FRB, assuming power-law spectra with $\Gamma=2$ and $\Gamma=4$. Limits on the average flux over $50 \mathrm{~ms}$ are also shown, derived by combining the data for the five FRBs. These limits are also valid for shorter integration time windows.

\begin{tabular}{lrcc}
\hline $\begin{array}{l}\text { FRB MJD } \\
(\mathrm{d})\end{array}$ & $\begin{array}{c}E_{0} \\
(\mathrm{GeV})\end{array}$ & $\begin{array}{c}\text { Int. flux UL }(\Gamma=2) \\
\left(10^{-7} \mathrm{~cm}^{-2} \mathrm{~s}^{-1}\right)\end{array}$ & $\begin{array}{c}\text { Int. flux UL }(\Gamma=4) \\
\left(10^{-7} \mathrm{~cm}^{-2} \mathrm{~s}^{-1}\right)\end{array}$ \\
\hline 57799.98 & 100 & 5.7 & 9.3 \\
& 400 & 2.9 & 3.1 \\
57806.96 & 1000 & 2.5 & 2.2 \\
& 100 & 5.7 & 9.3 \\
57806.98 & 400 & 2.9 & 3.1 \\
& 1000 & 2.5 & 2.2 \\
57808.00 & 100 & 5.6 & 10 \\
& 400 & 2.6 & 2.9 \\
& 1000 & 2.1 & 1.7 \\
57814.95 & 100 & 5.6 & 2.6 \\
& 400 & 2.2 & 1.4 \\
& 1000 & 1.7 & 8.5 \\
& 100 & 5.5 & 2.8 \\
Combined & 400 & 2.8 & 2.2 \\
& 1000 & 2.4 & 2.3 \\
& 100 & 1.2 & 0.59 \\
& 400 & 0.52 & 0.36 \\
\hline
\end{tabular}


Figure 2. Optical light curves covering $200 \mathrm{~ms}$ around the TOAs of the five radio bursts from FRB 121102 detected by the Arecibo telescope simultaneous with MAGIC data, for an integration window of $1 \mathrm{~ms}$. The vertical axis is proportional to the $U$-band flux. No significant excess is observed simultaneously with any of the five bursts. The noise level varies with the sky brightness.

no significant excess is detected simultaneously with any of the five FRB events. As discussed in Hassan et al. (2017), the sensitivity of the central pixel varies depending on the assumed duration of the signal. For integration times of $0.1,1,5$, and $10 \mathrm{~ms}$, the $5 \sigma$ sensitivity is $20,8.6,4.2$, and $3.2 \mathrm{mJy}$, respectively. The sensitivity 


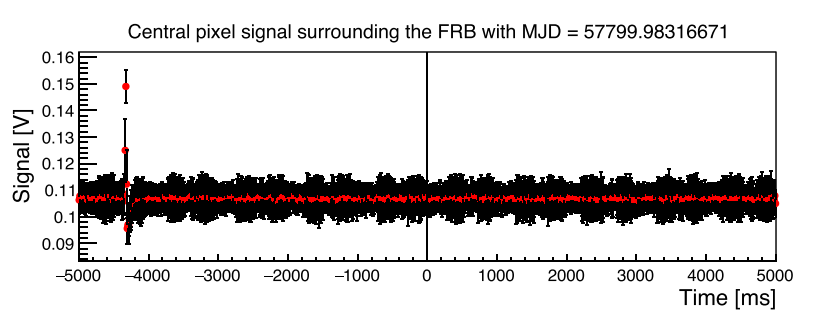

C. pixel signal surrounding the optical pulse, MJD $=57799.98311664$

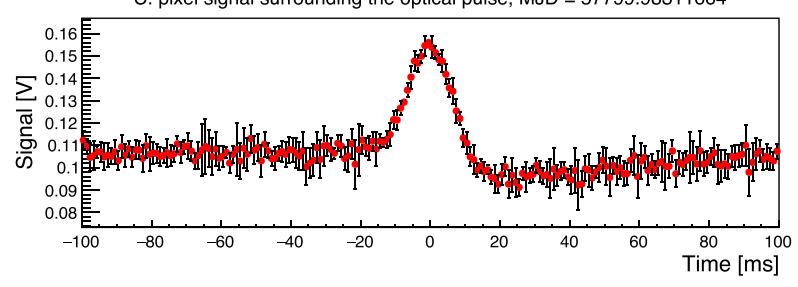

Figure 3. Top: Optical light curve covering $10 \mathrm{~s}$ around the first FRB in our sample, for an integration window of $10 \mathrm{~ms}$. A clear optical pulse is detected $4.3 \mathrm{~s}$ before the FRB. Bottom: Optical light curve covering $200 \mathrm{~ms}$ around the detected optical pulse, for an integration window of $1 \mathrm{~ms}$. The pulse is consistent with a background event. Note that the undershoot after the optical flash is caused by the central pixel readout electronics.

averaged over the five FRB events can also be derived by stacking the data around their TOAs, giving 12, 4.1, 2.3, and $1.7 \mathrm{mJy}$ for the same integration times as above.

As introduced in Section 2.2.2, the irreducible background of optical pulses within our OFF data sample hinders the search for optical bursts with arrival times offset from the radio TOAs. Nevertheless, due to the relatively low frequency of expected background events, searches for such optical pulses are worthwhile as long as the search window is sufficiently small. Thus, an unbiased search for 10-ms optical pulses around the radio TOAs was conducted, sequentially increasing the total search window around each FRB in equal logarithmic time steps (starting from $10 \mathrm{~ms}$, then $100 \mathrm{~ms}$, $1 \mathrm{~s}$, and so on). The number of trials for such a pulse search would correspond to the total number of 10-ms bins within the search window of all the FRB events scrutinized (e. g. if $1 \mathrm{~s}$ around each TOA was sampled, the total number of trials would correspond to $N=5$ TOAs $\times 1 \mathrm{~s} \times 100$ trials $\mathrm{s}^{-1}$ ).

A bright optical pulse with a peak brightness of $\sim 29 \mathrm{mJy}$ and full width at half-maximum of $12 \mathrm{~ms}$ was clearly detected $4.3 \mathrm{~s}$ before the first FRB in our sample (Fig. 3). No optical pulses are detected near the TOA of any other radio burst. Taking into account the frequency of pulses surviving analysis cuts within our OFF source data sample (a total of 17 within $15.5 \mathrm{~h}$ ), an optical pulse of this brightness is consistent with the observed background. The resulting chance probability is 1.5 percent post-trial. The time profile of this optical pulse is consistent with that of background pulses. For reference, the aforementioned Arecibo burst exhibited a radio peak brightness of $1.4 \mathrm{Jy}$ (see Table 1).

\section{DISCUSSION}

We have reported on $22 \mathrm{~h}$ of VHE gamma-ray and optical observations of FRB 121102 with MAGIC. Simultaneous radio observations were conducted with Arecibo for $8.9 \mathrm{~h}$, revealing a total of five radio bursts. We have derived constraints on the VHE and optical burst emission as well as the persistent VHE emission. Below we discuss some physical implications in light of potential scenarios for FRB 121102.

\subsection{Persistent VHE emission}

The origin of the persistent radio source associated with FRB 121102 remains unclear. One possibility is a pulsar wind nebula driven by a young rotation-powered neutron star or magnetar (Kashiyama \& Murase 2017; see however Waxman 2017), which may also emit persistent VHE gamma-rays via mechanisms analogous to known pulsar wind nebulae (Murase et al. 2016). Alternatively, it could be related to a BH with mass $\sim 10^{4}-10^{6} \mathrm{M}_{\odot}$ (Marcote et al. 2017), which may have associated persistent VHE emission similar to that observed from Sgr $\mathrm{A}^{\star}$ in the Galactic Center.

Fig. 1 shows the upper limits for persistent HE to VHE gammarays from FRB 121102. These are compared with SEDs of the Crab Nebula and $\mathrm{Sgr} \mathrm{A}^{\star}$, that have been scaled, respectively, by factors of $4 \times 10^{5}$ and $2 \times 10^{6}$ to match the observed radio luminosity of the persistent radio source associated with FRB 121102. Also shown in Fig. 1 is the effect of attenuation of the gamma-rays by $\gamma \gamma$ pair production interactions with the extragalactic background light (EBL), which is significant above $\sim 400 \mathrm{GeV}$ at the redshift of FRB 121102 (e.g. Domínguez et al. 2011).

The current upper limits for persistent gamma-ray emission lie $\sim 2-4$ orders of magnitude above such simple expectations based on luminosity scaling, and cannot provide significant constraints on the nature of FRB 121102.

\subsection{VHE burst emission associated with FRBs}

As shown in Table 3, the obtained upper limits on the burst-like VHE photon flux simultaneous with the FRBs are in the range $\sim(3-10)$ $\times 10^{-7} \mathrm{~cm}^{-2} \mathrm{~s}^{-1}$ above $100-400 \mathrm{GeV}$ over durations of $10 \mathrm{~ms}$. With the luminosity distance $d_{\mathrm{L}}=972 \mathrm{Mpc}$ for FRB 121102, this implies limits on the VHE luminosity per burst of $L_{\mathrm{VHE}} \lesssim(3-14)$ $\times 10^{49} \mathrm{erg} \mathrm{s}^{-1}$. Also, from the limits on the flux averaged over the FRBs during $50 \mathrm{~ms}$, the upper limit on the VHE radiation energy per burst can be roughly estimated as $\mathcal{E}_{\mathrm{VHE}} \lesssim(3-9) \times 10^{47} \mathrm{erg}$. Limits above $1 \mathrm{TeV}$ would be a factor $\sim 10$ less constraining due to the effect of attenuation by the EBL.

Such upper limits can provide a valuable test of the magnetar scenario by constraining the burst energy carried by re-accelerated electron-positron pairs (Lyubarsky 2014; Murase et al. 2016). If each FRB results from release of the magnetic free energy $\mathcal{E}_{B}$ trapped in the neutron star magnetosphere, a highly relativistic magnetized outflow will be launched. When such an outflow interacts with the slower nebula and its energy is dissipated, pre-existing non-thermal electrons and positrons are accelerated and emit synchrotron and inverse-Compton emission, which may be observable as a broadband flare from the radio to VHE bands. The energy dissipation time-scale is highly uncertain and may range from a few $\mathrm{ms}$ to much longer, depending on e.g. the Lorentz factor of the outflow (Murase et al. 2016). In the fast cooling regime where electrons and positrons cool within the dynamical time, the radiated energy at VHE can be as large as $\mathcal{E}_{\mathrm{VHE}} \sim 10^{47} \mathrm{erg}(\mathcal{C} / 10)^{-1}\left(\mathcal{E}_{B} / 10^{48} \mathrm{erg}\right)$, where $\mathcal{C}=O(10)$ is a factor that accounts for bolometric correction. Our results imply that the released magnetic energy may be constrained to be $\mathcal{E}_{\mathrm{B}} \lesssim 10^{48} \mathrm{erg}$ in the fast-cooling limit.

The current constraint on the energetics is not very stringent but can be significantly improved in the future. As the number $N_{\text {FRB }}$ of observed FRBs increases, the constraint would become tighter by a factor of about $N_{\mathrm{FRB}}$ in the background-free limit, or $N_{\mathrm{FRB}}^{1 / 2}$ if the background is non-negligible. For example, if the MAGIC telescopes could obtain similar limits for $N_{\mathrm{FRB}}=500$ repeating bursts from FRB 121102, the upper limit on the released energy 
will reach $\mathcal{E}_{\mathrm{B}} \lesssim 10^{46} \mathrm{erg}$, comparable to that of known magnetar hyper-flares (Popov \& Postnov 2013; Lyubarsky 2014). Such observations by current Cherenkov telescopes may be feasible over a period of 5-10 yr, given the frequency of bursts observed from FRB 121102 during some periods that can be as large as 18 bursts in 30 min Gajjar et al. (2018). If other sources of repeating FRBs are found that are more nearby, the constraints could be tightened by a factor $\sim d_{\mathrm{L}}^{2}$ at $\sim 100 \mathrm{GeV}$, and by a larger factor at higher energies by virtue of the reduced EBL attenuation. Further drastic improvements can be expected with future radio and VHE observatories, such as the Square Kilometer Array ${ }^{2}$ and the Cherenkov Telescope Array. ${ }^{3}$ Thus, VHE observations simultaneous with radio bursts provide a potentially powerful test of the magnetar model for FRB progenitors.

\subsection{Optical burst emission associated with FRBs}

No optical bursts on ms time-scales coincident with radio bursts have been detected so far. We constrain the optical flux to be below 4.1 and $1.7 \mathrm{mJy}$ at $5 \sigma$ confidence level for 1 and $10-\mathrm{ms}$ time windows around the radio TOAs, respectively. For optical bursts unassociated with radio bursts, limits of 8.6 and $3.2 \mathrm{mJy}$ are obtained for 1 and 10-ms duration, respectively. We note that a significant fraction of individual bursts from FRB 121102 are observed to emit within a relatively narrow frequency range in the radio domain (Law et al. 2017). Thus, correlations between radio and optical bursts may possibly be weak. Compared to previously reported upper limits on the fluence at $767 \mathrm{~nm}$ of $46 \mathrm{mJy}$ ms (Hardy et al. 2017), our upper limits provide the most stringent constraints on the putative optical burst emission of FRB 121102 to date. This can be contrasted with the Crab Pulsar, for which coincident radio and optical pulses have been reported (Shearer et al. 2003) with a radio-to-optical flux density slope (energy index) of $\alpha \sim-0.2$ (see e.g. Lyne \& GrahamSmith 2005). For FRB 121102, the optical upper limits presented here provide a strong constraint on this slope of $\alpha \lesssim-0.32$.

As shown in Fig. 3, we have detected an optical pulse with peak brightness of $28.9 \mathrm{mJy}$ that arrived $\sim 4.3 \mathrm{~s}$ before a $1.4-\mathrm{Jy}$ radio burst, for which the TOA has been corrected for dispersion at infinite frequency and topocentric time. Optical pulses preceding radio pulses have been previously observed in giant radio pulses from the Crab Pulsar (Shearer et al. 2003; Strader et al. 2013). If we assume that the optical pulse and radio burst of FRB 121102 are physically connected, it would point to a slope between the optical and radio flux of $\alpha \sim-0.3$, similar to but slightly steeper than that observed in the Crab pulses $(\alpha \sim-0.2)$. However, the detected optical pulse is compatible with the time profile and brightness of known background signals such as meteors and we cannot ascertain its origin. Further observations by MAGIC and other high timeresolution optical telescopes will provide stronger tests of potential optical burst emission from FRB 121102.

\section{CONCLUSIONS}

We have conducted simultaneous radio, optical, and VHE observations of FRB 121102 with the Arecibo and MAGIC telescopes, in order to search for burst emission on millisecond time-scales at these wavelengths. For the first time, we constrain the VHE and optical burst emission simultaneous with FRBs, five of which were detected

\footnotetext{
${ }^{2}$ https://www.skatelescope.org/

${ }^{3}$ https://www.cta-observatory.org/
}

during our campaign. We obtain limits of $0.5 \times 10^{-7} \mathrm{~cm}^{-2} \mathrm{~s}^{-1}$ for the average flux above $100 \mathrm{GeV}$ during the duration of the five FRBs, with interesting future implications for constraining some FRB models involving magnetars. We also set limits of $8.6 \mathrm{mJy}$ for the $U$-band flux during 1-ms intervals around the FRB arrival times, the strongest such constraints to date. We also obtain limits on the persistent VHE emission comparable to that already reported by Bird et al. (2017), which are still 2-4 orders of magnitude above simple expectations based on scaling the SEDs of well known sources such as the Crab Nebula and Sgr $A^{\star}$.

The optical pulse observed $4.3 \mathrm{~s}$ before the first FRB detected by Arecibo during our campaign cannot be unambiguously associated with FRB 121102 (being at 2.2 $\sigma$ confidence level). This is consistent with the fact that no other bright optical pulse has been found within a few seconds of the other four FRBs. However, it is worth noting that the spectra and time profiles of FRBs are known to be extremely variable from burst to burst. It is possible that only a limited number of these bursts are sufficiently bright to be detectable at higher frequencies, encouraging more searches for optical pulses.

\section{ACKNOWLEDGEMENTS}

We would like to thank the Instituto de Astrofísica de $\mathrm{Ca}$ narias for the excellent working conditions at the Observatorio del Roque de los Muchachos in La Palma. The financial support of the German BMBF and MPG, the Italian INFN and INAF, the Swiss National Fund SNF, the ERDF under the Spanish MINECO (FPA2015-69818-P, FPA2012-36668, FPA2015-68378-P, FPA2015-69210-C6-2-R, FPA2015-69210-C64-R, FPA2015-69210-C6-6-R, AYA2015-71042-P, AYA201676012-C3-1-P, ESP2015-71662-C2-2-P, CSD2009-00064), and the Japanese JSPS and MEXT is gratefully acknowledged. This work was also supported by the Spanish Centro de Excelencia 'Severo Ochoa' SEV-2012-0234 and SEV-2015-0548, and Unidad de Excelencia 'María de Maeztu' MDM-2014-0369, by the Croatian Science Foundation (HrZZ) Project IP-2016-06-9782 and the University of Rijeka Project 13.12.1.3.02, by the DFG Collaborative Research Centers SFB823/C4 and SFB876/C3, the Polish National Research Centre grant UMO-2016/22/M/ST9/00382 and by the Brazilian MCTIC, CNPq, and FAPERJ. SI thanks support from JSPS KAKENHI Grant Number JP17K05460. The work of KM is partially supported by Alfred P. Sloan Foundation and NSF grant No. PHY-1620777.

\section{REFERENCES}

Abdo A. A. et al., 2009, ApJS, 183, 46

Aharonian F. et al., 2009, A\&A, 503, 817

Aleksić J. et al., 2016a, Astropart. Phys., 72, 61

Aleksić J. et al., 2016b, Astropart. Phys., 72, 76

Bassa C. G. et al., 2017, ApJ, 843, L8

Bird R., for the VERITAS Collaboration, 2017, proceeding, 35th International Cosmic Ray Conference (ICRC 2017), Bexco, Busan, Korea (arXiv:1708.04717)

Borla Tridon D., for the MAGIC Collaboration, 2009, proceeding, 31st International Cosmic Ray Conference (ICRC 2009), Lódz, Poland (arXi v:0906.5448)

Chatterjee S. et al., 2017, Nature, 541, 58

Connor L., Sievers J., Pen U. L., 2016, MNRAS, 458, L19

Cordes J. M., Wasserman I., 2016, MNRAS, 457, 232

Domínguez A. et al., 2011, MNRAS, 410, 2556

Gajjar V. et al., 2018, ApJ, 863, 2

Ghisellini G., Locatelli N., 2018, A\&A, 613, A61 
Hardy L. K. et al., 2017, MNRAS, 472, 2800

Hassan T., for the MAGIC Collaboration, 2017, proceeding, 35th International Cosmic Ray Conference (ICRC 2017), Bexco, Busan, Corea (arXiv:1708.07698)

Inoue S., 2004, MNRAS, 348, 999

Ioka K., 2003, ApJ, 598, L79

Kashiyama K., Murase K., 2017, ApJ, 839, L3

Katz J. I., 2018, Fast radio bursts, Progress in Particle and Nuclear Physics, 103 (arXiv:1804.09092)

Kumar P., Lu W., Bhattacharya M., 2017, MNRAS, 468, 2726

Law C. J. et al., 2017, ApJ, 850, 76

Lorimer D. R., Bailes M., McLaughlin M. A., Narkevic D. J., Crawford F., 2007, Science, 318, 777

Lucarelli F. et al., 2008, Nucl. Instrum. Methods Phys. Res., 589, 415

Lunnan R. et al., 2014, ApJ, 787, 138

Lyne A. G., Graham-Smith F., 2005, Pulsar Astronomy, 3rd ed. Cambridge astrophysics series, Cambridge University Press, Cambridge, UK

Lyubarsky Y., 2014, MNRAS, 442, L9

Lyutikov M., 2002, ApJ, 580, L65

Lyutikov M., Burzawa L., Popov S. B., 2016, MNRAS, 462, 941

Marcote B. et al., 2017, ApJ, 834, L8

Meyer M., Horns D., Zechlin H. S., 2010, A\&A, 523, A2

Michilli D. et al., 2018, Nature, 553, 182

Murase K., Kashiyama K., Mészáros P., 2016, MNRAS, 461, 1498

Patrignani C., Particle Data Group, 2016, Chin. Phys. C, 40, 100001

Pen U. L., Connor L., 2015, ApJ, 807, 179

Petroff E. et al., 2016, Publ. Astron. Soc. Aust., 33, e045

Popov S. B., Postnov K. A., 2013, preprint (arXiv:1307.4924)

Rane A., Lorimer D., 2017, J. Astrophys. Astron., 38, 55

Rolke W. A., López A. M., Conrad J., 2005, Nucl. Instrum. Methods Phys. Res. A, 551, 493

Scholz P. et al., 2016, in AAS/High Energy Astrophysics Division. p. 105.03

Scholz P. et al., 2017, ApJ, 846, 80

Shearer A., Stappers B., O'Connor P., Golden A., Strom R., Redfern M., Ryan O., 2003, Science, 301, 493

Spitler L. G. et al., 2014, ApJ, 790, 101

Spitler L. G. et al., 2016, Nature, 531, 202

Strader M. J. et al., 2013, ApJ, 779, L12

Tendulkar S. P., Kaspi V. M., Patel C., 2016, ApJ, 827, 59

Tendulkar S. P. et al., 2017, ApJ, 834, L7

Thornton D. et al., 2013, Science, 341, 53

Vieyro F. L., Romero G. E., Bosch-Ramon V., Marcote B., del Valle M. V., 2017, A\&A, 602, A64

Waxman E., 2017, ApJ, 842, 34

Zanin R., for the MAGIC Collaboration, 2013, proceedings, 33rd International Cosmic Ray Conference (ICRC2013): Rio de Janeiro, Brazil, 2013. p. 0773

Zhang B., Li K., 2018, ApJ, 854, 123

Zhang B. B., Zhang B., 2017, ApJ, 843, L13

${ }^{1}$ Instituto de Astrofísica de Canarias, E-38200 La Laguna, and Universidad de La Laguna, Dpto. Astrofísica, E-38206 La Laguna, Tenerife, Spain

${ }^{2}$ Japanese MAGIC Consortium: ICRR, The University of Tokyo, 277-8582 Chiba, Japan; Department of Physics, Kyoto University, 606-8502 Kyoto,
Japan; Tokai University, 259-1292 Kanagawa, Japan; RIKEN, 351-0198

Saitama, Japan

${ }^{3}$ Università di Udine, and INFN Trieste, I-33100 Udine, Italy

${ }^{4}$ National Institute for Astrophysics (INAF), I-00136 Rome, Italy

${ }^{5}$ ETH Zurich, CH-8093 Zurich, Switzerland

${ }^{6}$ Università di Padova and INFN, I-35131 Padova, Italy

${ }^{7}$ Technische Universität Dortmund, D-44221 Dortmund, Germany

${ }^{8}$ Croatian MAGIC Consortium: University of Rijeka, 51000 Rijeka, University of Split - FESB, 21000 Split, University of Zagreb - FER, 10000 Zagreb, University of Osijek, 31000 Osijek and Rudjer Boskovic Institute, 10000 Zagreb, Croatia

${ }^{9}$ Saha Institute of Nuclear Physics, HBNI, 1/AF Bidhannagar, Salt Lake, Sector-1, Kolkata 700064, India

${ }^{10}$ Max-Planck-Institut für Physik, D-80805 München, Germany

${ }^{11}$ Unidad de Partículas y Cosmología (UPARCOS), Universidad Complutense, E-28040 Madrid, Spain

${ }^{12}$ University of Łódź, Department of Astrophysics, PL-90236 Łódź, Poland

${ }^{13}$ Deutsches Elektronen-Synchrotron (DESY), D-15738 Zeuthen, Germany

${ }^{14}$ Humboldt University of Berlin, Institut für Physik D-12489 Berlin Germany

${ }^{15}$ Dipartimento di Fisica, Università di Trieste, I-34127 Trieste, Italy

${ }^{16}$ Institut de Física d'Altes Energies (IFAE), The Barcelona Institute of Science and Technology (BIST), Bellaterra, E-08193 Barcelona, Spain

${ }^{17}$ Università di Siena and INFN Pisa, I-53100 Siena, Italy

${ }^{18}$ Università di Pisa, and INFN Pisa, I-56126 Pisa, Italy

${ }^{19}$ Port d'Informació Científica (PIC) Bellaterra, E-08193 Barcelona, Spain

${ }^{20}$ Universität Würzburg, D-97074 Würzburg, Germany

${ }^{21}$ Finnish MAGIC Consortium: Tuorla Observatory and Finnish Centre of Astronomy with ESO (FINCA), University of Turku, Vaisalantie 20, FI-21500 Piikkiö, Astronomy Division, University of Oulu, FIN-90014 University of Oulu, Finland

${ }^{22}$ Departament de Física, and CERES-IEEC, Universitat Autónoma de Barcelona, E-08193 Bellaterra, Spain

${ }^{23}$ Institute for Nuclear Research and Nuclear Energy, Bulgarian Academy of Sciences, BG-1784 Sofia, Bulgaria

${ }^{24}$ Universitat de Barcelona, ICC, IEEC-UB, E-08028 Barcelona, Spain

${ }^{25}$ INAF-Trieste and Department of Physics \& Astronomy, University of Bologna

${ }^{26}$ Joint Institute for VLBI ERIC, Postbus 2, 7990 AA Dwingeloo, the Netherlands

${ }^{27}$ Max-Planck-Institut für Radioastronomie, Auf dem Hügel 69, D-53121 Bonn, Germany

${ }^{28}$ ASTRON, Netherlands Institute for Radio Astronomy, Postbus 2, NL-7990 AA Dwingeloo, the Netherlands

${ }^{29}$ Anton Pannekoek Institute for Astronomy, University of Amsterdam, Science Park 904, NL-1098 XH Amsterdam, the Netherlands

${ }^{30}$ Department of Physics, the University of Tokyo, Bunkyo, Tokyo 113-0033, Japan

${ }^{31}$ Department of Physics, the Pennsylvania State University, University Park, PA 16802, USA

${ }^{32}$ National Astronomy and Ionosphere Center, Arecibo Observatory, Puerto Rico 00612, USA

This paper has been typeset from a $\mathrm{T}_{\mathrm{E}} \mathrm{X} / \mathrm{L} \mathrm{T}_{\mathrm{E}} \mathrm{X}$ file prepared by the author. 